\title{
The E2F family as potential biomarkers and therapeutic targets in colon cancer
}

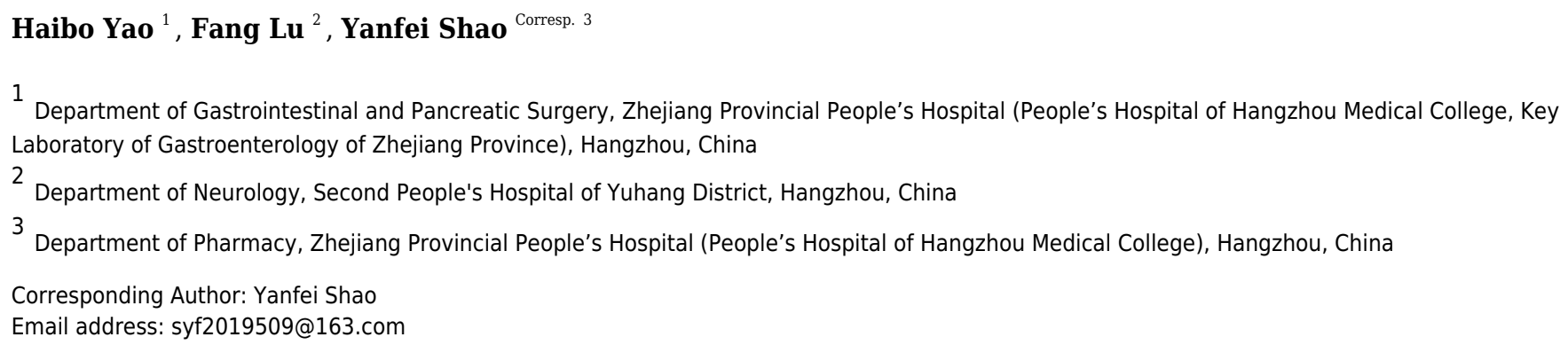

Background: The E2F family is a group of genes encoding a series of transcription factors in higher eukaryotes and participating in the regulation of cell cycle and DNA synthesis in mammals. This study was designed to investigate the role of E2F family in colon cancer. Methods: In this study, the transcriptional levels of E2F1-8 in patients with colon cancer from GEPIA was examined. Meanwhile, the immunohistochemical data of the 8 genes were also obtained in the The Human Protein Atlas website. Additionally, we re-identified the mRNA expression levels of these genes via real time PCR. Furthermore, the association between the levels of E2F family and stage plot as wells overall survival of patients with colon cancer were analyzed. Results: We found that the mRNA and protein levels of E2F1, E2F2, E3F3, E2F5, E2F7 and E2F8 were significantly higher in colon cancer tissues than in normal colon tissues while the expression levels of E2F4 and E2F6 displayed no significant difference between colon cancer tissues and normal tissues. Additionally, E2F3, E2F4, E2F7 and E2F8 were significantly associated with the stages of colon cancer. The Kaplan-Meier Plotter showed that the high levels of E2F3 conferred a worse overall survival and disease free survival of patients with colon cancer. And high level of E2F4 resulted in a worse overall survival. Conclusion: Our study implied that E2F3, E2F4, E2F7 and E2F8 are potential targets of precision therapy for patients with colon cancer while E2F1, E2F2, E3F3, E2F5, E2F7 and E2F8 are potential biomarkers for the diagnosis of colon cancer. 
1

\section{Title Page}

3

4 Manuscript Title:

5 The E2F family as potentialbiomarkers and therapeutic targets in colon cancer

6 Running Title:

7 The E2F in colon cancer

8 Authors:

$9 \quad$ Haibo $\mathrm{Yao}^{1}$, Fang $\mathrm{Lu}^{2}$, Yanfei $\mathrm{Shao}^{3, *}$

10 Affiliations:

11 'Department of Gastrointestinal and Pancreatic Surgery, Zhejiang Provincial People's Hospital (People's

12 Hospital of Hangzhou Medical College, Key Laboratory of Gastroenterology of Zhejiang Province), Hangzhou

13 310014, China

$14{ }^{2}$ Department of Neurology, Second People's Hospital of Yuhang District, Hangzhou 310014, China

$15{ }^{3}$ Department of Pharmacy, Zhejiang Provincial People's Hospital (People's Hospital of Hangzhou Medical

16 College), Hangzhou 310014, China

17 *Correspondence to: Yanfei Shao, Department of Pharmacy, Zhejiang Provincial People's Hospital (People's

18 Hospital of Hangzhou Medical College), 158 Shangtang Road, Hangzhou 310014, China; Tel: +86-

19 13588702943, Email: syf2019509@163.com

20 Acknowledgements:

21 This study was supported by Natural Science Foundation of Zhejiang Province (LQ19H160013).

22 All conflict of interest disclosures:

23 None. 


\section{Abstract}

27 Background: The E2F family is a group of genes encoding a series of transcription factors in higher eukaryotes and participating in the regulation of cell cycle and DNA synthesis in mammals. This study was designed to investigate the role of E2F family in colon cancer.

Methods: In this study, the transcriptional levels of E2F1-8 in patients with colon cancer from GEPIA was examined. Meanwhile, the immunohistochemical data of the 8 genes were also obtained in the The Human Protein Atlas website. Additionally, we re-identified the mRNA expression levels of these genes via real time PCR. Furthermore, the association between the levels of E2F family and stage plot as wells overall survival of patients with colon cancer were analyzed.

Results: We found that the mRNA and protein levels of E2F1, E2F2, E3F3, E2F5, E2F7 and E2F8 were significantly higher in colon cancer tissues than in normal colon tissues while the expression levels of E2F4 and E2F6 displayed no significant difference between colon cancer tissues and normal tissues. Additionally, E2F3, E2F4, E2F7 and E2F8 were significantly associated with the stages of colon cancer. The Kaplan-Meier Plotter showed that the high levels of E2F3 conferred a worse overall survival and disease free survival of patients with colon cancer. And high level of E2F4 resulted in a worse overall survival.

Conclusion: Our study implied that E2F3, E2F4, E2F7 and E2F8 are potential targets of precision therapy for patients with colon cancer while E2F1, E2F2, E3F3, E2F5, E2F7 and E2F8 are potential biomarkers for the diagnosis of colon cancer.

\section{Key words colon cancer; E2F family; prognosis; biomarkers; bioinformatics}




\section{Introduction}

The E2F family is a group of genes that encode a family of transcription factors in higher eukaryotes[1], which are currently subdivided into 2 groups based on their functional characteristics: namely transcriptional activators including E2F1, E2F2, and E2F3a and transcriptional repressors including E2F3b, E2F4, E2F5, E2F6, E2F7 and E2F8 [2]. The E2F family was originally regarded as a cellular activity that is required for the early region 1A transforming protein of adenovirus to mediate the transcriptional activation of the viral E2 promoter[3]. Subsequent studies unveiled that E2F family also controls the transcription of cellular genes that are responsible for cell division[4]. The expression pattern of E2F activators is abnormal in multiple human malignancies, such as ovarian cancer[5], breast cancer[6], bladder cancer[7], prostate cancer[8], lung adenocarcinoma[9] and colon cancer[10].

Colon cancer is a common malignancy, which is currently ranked as the third most prevalent cancer and the third leading cause of cancer death in the United States[10]. Despite that considerable advancements in diagnostic and treatment methods, the 5-year overall survival rate of colon cancer remains no more than $21 \%[11]$. Hence, novel biomarkers and potential therapeutic targets should be screened to enhance prognosis and individualized treatments in patients with colon cancer. Of the 8 members in E2F family, many genes have been disclosed to participate in the development of colon cancer in vitro and in vivo. For instance, E2F1 could upregulate the expression of c-Myc and p14ARF, therefore inducing apoptosis in colon cancer cells[12]. E2F2 was also proved to act as a potential new molecular biomarker for colon carcinogenesis[13]. But to the best of our knowledge, bioinformatics analysis has yet been applied to investigate the roles of E2F gene family in colon cancer. Based on the analyses of thousands of gene expression or variation in copy numbers published online, we analyzed and validated the expression and different E2F transcription factors in patients with colon cancer in detail to explore the expression patterns, potential functions, and distinct prognostic values of transcription factors in colon cancer.

\section{Methods}

\subsection{Ethics statement}

Our study was conducted based on the principles expressed in the Declaration of Helsinki, and approved 
75

76

77

78

by the Academic Committee of Zhejiang Provincial People's Hospital (Ethical Application Ref: IR2019001002).

The datasets mentioned in our study were retrieved from the free and publicly available database, indicating that it was confirmed that all written informed consent was acquired.

\subsection{Comparison of the hub genes expression level}

GEPIA is a newly developed interactive web server for analyzing the RNA sequencing expression data including 9736 tumors and 8587 normal samples from the TCGA database and the GTEx projects in a standard processing manner[14]. GEPIA provides customizable functions including tumor/normal differential expression analysis, profiling according to cancer types or pathological stages, patient survival analysis, similar gene detection, correlation analysis, and dimensionality reduction analysis. In present study, we mainly employed the boxplot to visualized the expression of E2F genes in colon cancer tissues and adjacent tissues. Additionally, GEPIA was also employed to provide the transcripts per million (TPM) of E2F genes to display their relative expression level.

\subsection{The overall survival (OS) and stage plot of E2F}

Similarly, we used the GEPIA database to get the overall survival (OS) and stage plot information of E2F genes of 135 patients with high level of E2Fs and 135 patients with low levels of E2Fs. The log rank P value and hazard ratio (HR) with 95\% confidence intervals were showed on the plot. $\mathrm{P}<005$ was statistically significant.

\subsection{Immunohistochemistry staining}

The Human Protein Atlas (HPA, https://www.proteinatlas.org/) is a Swedish-based program initiated in 2003 with the aim to map all human proteins in cells, tissues, and organs using the integration of various omics technologies, including antibody-based imaging, mass spectrometry-based proteomics, transcriptomics, and systems biology. By acquiring immunohistochemical data of patients with or without colon cancer based on HPA, we further verified the expression of E2F genes.

\subsection{Gene Ontology and KEGG Pathway Analysis of E2F genes}

Gene Ontology (GO) analysis is a common framework which can annotate genes and gene products including functions of Cellular Components (CC), Biological Pathways (BP) and Molecular Function (MF). Kyoto Encyclopedia of Genes and Genomes (KEGG) contains a set of genomes and biological pathways related 
102

103

with disease and drugs online database, which essentially is a resource for systematic understanding of biological system and certain high-level genome functional information. The Database for Annotation, Visualization and Integrated Discovery (DAVID, http://david.ncifcrf.gov, version DAVID 6.7) is an online bioinformatics database. It has covered a great many biological data and relevant analysis tools, then provide tools for the biological function annotation information for plenty of genes or proteins. $\mathrm{P}<0.05$ was considered as the cutoff criterion with significant difference. We could visualize the key biological processes, molecular functions, cellular components and pathways of DEGs by using DAVID online database. And further the scatter plot was performed by ImageGP（http://www.ehbio.com/ImageGP/index.php/Home/Index/index.html ） according to the results of GO and KEGG pathway.

\subsection{Quantitative Real-Time PCR (qPCR)}

Thirty-two pairs of colon tissues and surrounding adjacent tissues were obtained from patients diagnosed with colon cancer in Zhejiang Provincial People's Hospital. Total RNA $(1 \mu \mathrm{g})$ was isolated using the TRIzol (Invitrogen, Carlsbad, CA, USA) kit, the concentrations and purities of which were quantified using an ultraviolet spectrophotometer. After that, cDNA was generated from RNA via reverse-transcribing using Transcriptor First Strand cDNA Synthesis Kit (Roche, USA) and followed real-time PCR was administrated using LightCycler 480 SYBR Green Master Mix (Roche Diagnostics GmbH)[32]. The expression levels of E2F genes were normalized to GAPDH. Relative mRNA expression levels were analyzed by the $2^{-\Delta \Delta}$ cycle threshold (CT) method. The primer sequences are displayed in Table 1.

\subsection{TCGA data and cBioPortal}

The Cancer Genome Atlas possessed both pathological data and sequencing of 30 different cancers. The liver colon carcinoma (TCGA, Provisional) dataset involving data from 618 samples with pathology reports was chosen for comprehensive analyses of E2Fs using cBioPortal. The genomic profiles included mutations, putative copy-number alterations (CNA) from GISTIC, mRNA expression z-scores (RNASeq V2 RSEM) and protein expression Z-scores (RPPA).

\subsection{Statistical analysis}

All values were reported as means $\pm \mathrm{SD}$. A paired, two-sided Student's t test was used to compare differences between two groups. Statistical significance was analyzed by SPSS 19.0 software. Differences 
129

130

131

132

133

134

135

136

137

138

139

140

141

142

143

were considered significant when $\mathrm{P}<0.05$.

\section{Results}

\subsection{Expression levels of E2F genes in patients with colon cancer}

By analyzing 349 normal colon tissues and 275 colon cancer tissues in GEPIA online website, we found the mRNA expression levels of E2F1, E2F2, E2F3, E2F5, E2F7 and E2F8 were significantly higher in patients with colon cancer than normal control $(\mathrm{P}<0.05)$ while E2F4 and E2F6 displayed no significant difference between colon cancer group and control group $(\mathrm{P}>0.05)$ (Figure1 A-H). Meanwhile, the above results were further identified using Oncomine database (Supplementary Figure1).

\subsection{Transcripts per million (TPM) of E2F genes}

Transcripts per million (TPM) serves as a measurement of the proportion of transcripts in the pool of RNA. In our study, the higher TPM levels of E2F1, E2F2, E2F3, E2F5, E2F7 and E2F8 in colon cancer group were observed $(\mathrm{P}<0.05)$, suggesting that the above 6 genes possessed more transcripts in colon cancer tissues. Consistent with the mRNA levels of E2F4 and E2F6, no significant difference was found between the 2 groups $(\mathrm{P}>0.05)$ (Figure2).

\subsection{Correlation between E2F genes expression and tumor stage in patients with colon cancer}

Subsequently, we analyzed the correlation between E2F genes expression and tumor stage in patients with colon cancer based on GEPIA online website. The results demonstrated that the expression levels of E2F3, E2F4, E2F7 and E2F8 displayed significant correlation with the tumor stage in patients with colon cancer while other members in E2F family in normal group and tumor group did not significantly differ (Figure 3A-H).

\subsection{GO and KEGG enrichment Analysis}

The results (Table2) from GO term enrichment analysis varied from expression levels and GO classification of the DEGs. By analyzing GO enrichment of these E2F genes via DAVID, we found that the E2F genes in BP were mainly enriched in transcription, DNA-templated, positive regulation of DNA endoreduplication, hepatocyte differentiation, negative regulation of cytokinesis and chorionic trophoblast cell differentiation. As for $\mathrm{CC}$, the $\mathrm{E} 2 \mathrm{~F}$ genes were principally enriched in transcription factor complex. MF analysis uncovered that the E2F genes were mainly enriched in core promoter binding, transcription factor activity, sequence-specific DNA 
binding, DNA binding and transcription corepressor activity.

To acquire a more comprehensive information regarding to the critical pathways of those selected

DEGs, KEGG pathways analysis were also carried out via DAVID. The results in Table3 unveiled the most vital KEGG pathways of the E2F genes, which were mainly enriched in cell cycle and various malignant tumors including bladder cancer, non-small cell lung cancer and pancreatic cancer.

\subsection{Correlation between E2F genes expression and overall survival in patients with colon cancer}

Meanwhile, we further explored the potential association between the expression levels of E2F genes and the overall survival of patients with colon cancer (Figure 4A-H). The Kaplan-Meier showed that E2F3 and E2F4 displayed significantly correlation with the overall survival of patients with colon cancer. To be more specific, the high levels of E2F3 and E2F4 may contribute to worse prognosis of colon cancer $(\mathrm{P}<0.05)$. Also, we explored the disease free survival of these genes in patients with colon cancer. The results (Supplementary Figure2) showed that lower level of E2F3 may contribute to better disease free survival in patients with colon cancer. Furthermore, we analyzed the E2F alterations by using the cBioPortal online tool for colon cancer (http://www.cbioportal.org/). E2Fs were altered in 109 samples out of 618 patients with lung adenocarcinoma (17.7\%). Two or more alterations were detected in almost one fifth of the samples (Supplementary Figure3).

\subsection{The immunohistochemical staining of E2F genes}

Additionally, we detected the protein expression levels of E2F genes based on the Human Protein Atlas online database. In accordance with the mRNA expression levels obtained from GEPIA, the protein expression of E2F1, E2F2, E2F3, E2F5, E2F7 and E2F8 were significantly higher in carcinoma tissues (Figure 5).

\subsection{Re-identification of the mRNA expression levels of E2F genes}

To further valid the expression patterns of E2F genes in colon cancer, we detected the mRNA levels of E2F genes in 32 patients with colon cancer using real time PCR. The results (Figure 6) showed that the mRNA levels of E2F1, E2F2, E2F3, E2F5, E2F7 and E2F8 were significantly lower in adjacent tissues compared with those in carcinoma tissues (Figure 6).

\section{Discussion}

The dysregulation of E2F genes has been reported in a great many cancers, including gastric cancer, lung 
cancer, hepatocellular carcinoma, pancreatic cancer and colon cancer[15][33,34]. Further bioinformatics analysis of colon cancer has to be further performed although the roles of E2F factors in the tumorigenesis and prognosis of certain cancers have been elaborated. To our knowledge, our study was the first time to investigate the mRNA and protein expression, and prognostic values of different E2F genes in patients with colon cancer. We sincerely hope our bioinformatics analysis could contribute to extra proof, optimize treatment strategy, and improve the accuracy of diagnosis and prognosis for patients with colon cancer.

E2F1 is the most explored in colon cancer among the 8 genes of E2F family. E2F1 regulates G1/S-phase transition of cell cycle by transactivating multiple genes including chromosomal DNA replication and its own promoter[16]. E2F1 itself could be regulated in a cell cycle-dependent manner, mainly through temporal association with retinoblastoma, a pocket protein family member. In turn, pocket proteins could be regulated by phosphorylated cyclin-dependent kinase[17]. The transcription of the thymidylate synthase gene was regulated by E2F1 in primary colon cancer specimens, the regulatory pattern of which from E2F1 to thymidylate synthase may be highly conserved during malignant progression of colon cancer[18]. Additionally, in p53 deficient human colon cancer cells, Mdm2 inhibition could trigger cell apoptosis by activating E2F1-and p73- mediated protein expression of Siva-1 and PUMA[19]. In our study, we found that the mRNA and protein expression of E2F1 was significantly higher in colon carcinoma, however, the expression level of which did not affect tumor stage and overall survival, suggesting that E2F1 may just serve as a tumor "switch" to induce tumorigenesis.

E2F2 plays dual roles in the occurrence and development of tumor. On the one hand, E2F2 can indeed serve as an "activator" to increase the expression of its targets[20]. On the other hand, E2F2 can also be a suppressor by repressing cell cycle regulators to maintain quiescence and suppressing myc-induced proliferation and tumorigenesis[21].For example, E2F2 could suppress the proliferation of T lymphocytes[22]. In fact, the expression level of E2F2 was very low level in colon cancer and E2F2 inhibition could enhance proliferation and cell cycle of colon cancer cells by suppressing the expression of surviving and regulating the expression of C-MYC, CCNA2, MCM4[23] as well as CDK2 [24]. Our analysis unveiled that the mRNA and protein expression levels of E2F2 were significantly higher in colon cancer tissues. Similarly, the expression level of E2F2 was not associated with tumor stage and overall survival in patients with colon cancer.

E2F3 is critical for the transcriptional activation of various oncogenes controlling the rate of proliferation 
210 of both tumor and primary cells. For example, miR-125b could block the colonies form of bladder cancer cells

211 in vitro and to develop tumors in nude mice by targeting E2F3[25]. In colon cancer, E2F3 serves as a direct

212 target of miR-503 that is responsible for the proliferation and cell cycle distribution. The inhibition of E2F3 not 213 only suppressed proliferation but also triggered apoptosis and G0/G1 arrest in SW480 colon cancer cells[25]. In

214 our report, the expression of E2F3 in colon cancer tissues was higher than that in normal tissues. We also 215 disclosed that E2F2 expression was significantly correlated with tumor stage in patients with colon cancer. A high E2F2 expression was significantly correlated with poor survival in patients with LC.

217 E2F4 as well as its target cyclin A were significantly up-regulated and mostly nuclear in human colon tumor 218 cells compared with the corresponding benign epithelium[26]. The mutations of E2F4 also enhanced the capacity 219 of colon cancer cells to grow without anchorage, therefore giving rise to tumor progression[27]. In our study, 220 although there was no significant difference of the expression between colon cancer tissues and normal tissues, 221 the level of E2F4 was associated with tumor stage as well as overall survival in patients with colon cancer.

E2F5 was a bona fide target gene of miR-34a and the restoration of E2F5 significantly antagonized the suppression of colon cancer cell proliferation and invasion[28]. Our study demonstrated that the expression of E2F5 was significantly increased in colon cancer tissues, however, the level of which did not affect the tumor stage and survival rate of patients with colon cancer.

Up to now, there were no studies on the role of E2F6 in colon cancer published, which was consistent with our bioinformatic analysis, as evidenced by the undifferentiated expression levels of E2F6. Similarly, the expression levels of E2F6 had no effect on the tumor stage and overall survival of patients with colon cancer based on our findings.

The E2F7 gene, located at chromosome 12q21.2, contains 14 exons and acts as a tumor suppressor in the regulation of cell cycle progression[29]. A significant association between E2F7 missense variant rs3829295 and $\mathrm{CRC}$ susceptibility, especially in males, was found in a Chinese Han population[30]. Additionally, miR520a could regulate inflammatory reactions by targeting E2F7 in colon cancer[31]. Our findings disclosed that the expression of E2F3 had an association with colon cancer stage, meanwhile the expression of E2F7 in colon cancer tissues were significantly increased in patients with colon cancer. 
237 different expressions between normal tissues and colon cancer tissues, apart from its association with tumor

238 stage. Hence, more experiments should be performed to explore the potential roles of E2F8 in colon cancer.

\section{5. Conclusion}

240 Our studies indicated that the deregulation of E2F1, E2F2, E2F3, E2F5, E2F7 and E2F8 in colon cancer

241 tissues might play a vital role in colon cancer oncogenesis, which could be promising diagnostic biomarkers for

242 LUAD. In addition, the expression of E2F3, E2F4, E2F7 and E2F8 were significantly associated with tumor

243 stages and overall survival of the patients with colon cancer, suggesting that they may serve as potential

244 therapeutic targets for colon cancer. Altogether, we hope our study may be helpful to potential prognostic

245 markers for the improvement of colon cancer survival and prognostic accuracy in the future. 
249 Funding

250 This study was supported by Natural Science Foundation of Zhejiang Province (LQ19H160013). 


\section{References}

253

1. Trimarchi JM, Lees JA: Sibling rivalry in the E2F family. Nat Rev Mol Cell Biol 2002, 3(1):11-20.

254

2. Kwon JS, Everetts NJ, Wang X, Wang W, Della KC, Xing J, Yao G: Controlling Depth of Cellular Quiescence by an Rb-E2F Network Switch. Cell Reports 2017, 20(13):3223-3235.

3. Shen $Y$, Nar R, Fan AX, Aryan M, Hossain MA, Gurumurthy A, Wassel PC, Tang M, Lu J, Strouboulis J:

10. Tazawa $\mathrm{H}$, Tsuchiya $\mathrm{N}$, Izumiya $\mathrm{M}$, Nakagama $\mathrm{H}$ : Tumor-suppressive miR-34a induces senescence-like

8. Kaseb AO, Chinnakannu K, Chen D, Sivanandam A, Tejwani S, Menon M, Dou QP, Reddy PV: Androgen

6. Millour J, Olano ND, Horimoto Y, Monteiro L, Langer JK, Aligue R, Hajji N, Lam EWF: ATM and p53 Regulate

4. Stead E, White JR, Conn S, Goldstone S, Rathjen J, Dhingra U, Rathjen P, Walker D, Dalton S: Pluripotent cell 5. Reimer D, Sadr S, Wiedemair A, Goebel G, Concin N, Hofstetter G, Marth C, Zeimet AG: Expression of the E2F family of transcription factors and its clinical relevance in ovarian cancer. Annals of the New York Academy of Sciences 2010, 1091(1):270-281.

7. Santos M, Dueñas M, Garcíaescudero R, Alfaya B, Villacampa F, Saizladera C, Costa C, Oteo M, Duarte J, FOXM1 Expression via E2F in Breast Cancer Epirubicin Treatment and Resistance. Molecular Cancer Therapeutics 2011, 10(6):1046-1058.

Martínez V: In vivo disruption of an Rb-E2F-Ezh2 signaling loop causes bladder cancer. Cancer Research 2014, 74(22):6565-6577.

Receptor- and E2F-1-Targeted Thymoquinone Therapy for Hormone-Refractory Prostate Cancer. Cancer Research 2007, 67(16):7782-7788.

9. Chen L, Kurtyka CA, Welsh EA, Rivera JI, Engel BE, Muñozantonia T, Yoder SJ, Eschrich SA, Creelan BC, Chiappori AA: Early2 factor (E2F) deregulation is a prognostic and predictive biomarker in lung adenocarcinoma. Oncotarget 2016, 7(50):82254. growth arrest through modulation of the E2F pathway in human colon cancer cells. Proc Natl Acad Sci U S A 2007, 104(39):15472-15477.

11. Bagaria SP, Heckman MG, Diehl NN, Parker A, Wasif N: Delay to Colectomy and Survival for Patients Diagnosed with Colon Cancer. Journal of Investigative Surgery the Official Journal of the Academy of Surgical Research 2018, 33(3_suppl):1.

12. Elliott MJ, Yan BD, Yang H, Mcmasters KM: E2F-1 Up-Regulates c-Myc and p14ARF and Induces Apoptosis in Colon Cancer Cells. Clinical Cancer Research An Official Journal of the American Association for Cancer Research 2001, 7(11):3590-3597.

13. Nicolet C, Neuville A, Flaman JM, Gaub MP, Guenot D: E2F2 - a potential new molecular marker for colon carcinogenesis. European Journal of Cancer Supplements 2008, 6(9):51-52.

14. Li N, Li L, Chen Y: The Identification of Core Gene Expression Signature in Hepatocellular Carcinoma. Oxidative Medicine \& Cellular Longevity 2018, 2018(4):1-15.

15. Nevins JR: The Rb/E2F pathway and cancer. Human Molecular Genetics 2001, 10(7):699-703.

16. Jiang H, Martin V, Alonso M, Gomez-Manzano C, Fueyo J: RB-E2F1: molecular rheostat for autophagy and 291 apoptosis. Autophagy 2010, 6(8):1216. 
292 17. Vasavi I, Prathyusha AMVN, Ramesh M, Kumar KS, Naidu BP, Bramhachari PV, Yugandhar NM, Ganesh PRC:

293

294

295

296

297

298

299

300

301

302

303

304

305

306

307

308

309

310

311

312

313

314

315

316

317

318

319

320

321

322

323

324

325

326

327

328

329

330

331

332

17. Vasavi I, Prathyusha AMVN, Ramesh M, Kumar KS, Naidu
E2F1: Transcriptional Machinery in Colon Cancer. 2017.

18. Kasahara M, ., Takahashi Y, ., Nagata T, ., Asai S, ., Eguchi T, ., Ishii Y, ., Fujii M, ., Ishikawa K, . Thymidylate synthase expression correlates closely with E2F1 expression in colon cancer. Clinical Cancer Research An Official Journal of the American Association for Cancer Research 2000, 6(7):2707-2711.

19. Ray RM, Bhattacharya S, Johnson LR: Mdm2 inhibition induces apoptosis in p53 deficient human colon cancer cells by activating p73- and E2F1-mediated expression of PUMA and Siva-1. Apoptosis 2011, 16(1):35-44.

20. Infante A, Laresgoiti UR, J, Fullaondo A, Galan J, Diaz-Uriarte R, Malumbres M, Field S, Zubiaga A: E2F2 represses cell cycle regulators to maintain quiescence. Cell Cycle 2008, 7(24):3915-3927.

21. Johnson DG, James D: Putting the Oncogenic and Tumor Suppressive Activities of E2F into Context. Current Molecular Medicine 2006, 6(7):-.

22. Azkargorta M, Arizmendi JM, Elortza F, Alkorta N, Zubiaga AM, Fullaondo A: Differential proteome profiles in E2F2-deficient T lymphocytes. Proteomics 2010, 6(S1):S42-S50.

23. $\mathrm{Li}$ T, Luo W, LiU K, LV X, Xi T: miR-31 promotes proliferation of colon cancer cells by targeting E2F2. Biotechnology Letters 2015, 37(3):523.

24. Xanthoulis A, Tiniakos DG: E2F transcription factors and digestive system malignancies: How much do we know? World Journal of Gastroenterology Wjg 2013, 19(21):3189-3198.

25. Huang L, Luo J, Cai Q, Pan Q, Zeng H, Guo Z, Dong W, Huang J, Lin T: MicroRNA-125b suppresses the development of bladder cancer by targeting E2F3. International Journal of Cancer 2011, 128(8):1758-1769.

26. Garneau H, Paquin MC, Carrier JC, Rivard N: E2F4 expression is required for cell cycle progression of normal intestinal crypt cells and colorectal cancer cells. Journal of Cellular Physiology 2010, 221(2):350-358.

27. Marie-Christine P, Caroline L, Etienne L, Benjamin B, Nathalie R: Functional impact of colorectal cancerassociated mutations in the transcription factor E2F4. International Journal of Oncology 2013, 43(6):20152022.

28. Guifeng L, Yaling S, Shengli A, Sainan X, Xiaoli R, Dan Z, Pingxiang W, Wenting L, Yanqing D, Li L: MicroRNA34a targets FMNL2 and E2F5 and suppresses the progression of colorectal cancer. Experimental \& Molecular Pathology 2015, 99(1):173-179.

29. Carvajal LA, Hamard PJ, Tonnessen C, Manfredi JJ: E2F7, a novel target, is up-regulated by p53 and mediates DNA damage-dependent transcriptional repression. Genes \& Development 2012, 26(14):15331545.

30. Ai YG, Kan Z, Jin LX, Jin LH, Lan G: Identification of a Low-Frequency Missense Variant in E2F Transcription Factor 7 Associated with Colorectal Cancer Risk In A Chinese Population. Asian Pacific Journal of Cancer Prevention Apjcp 2017, 18(1):271-275.

31. Cui M, Zhang M, Zhang L, Wang J, Zhou S: MiR-520a regulates cancer-related inflammation by targeting E2F7, IRF2, and Rab22a in colon cancer. Journal of Gastroenterology \& Hepatology.

32. Xiao Y, Wu QQ, Duan MX, Liu C, Yuan Y, Yang Z, Liao HH, Fan D, Tang QZ. TAX1BP1 overexpression attenuates cardiac dysfunction and remodeling in STZ-induced diabetic cardiomyopathy in mice by regulating autophagy. Biochimica et Biophysica Acta (BBA) - Molecular Basis of Disease, 2018, 1864(5 Pt A).

33. Marco Vacante, Antonio Maria Borzì, Francesco Basile, Biomarkers in colorectal cancer: Current clinical 
333

334

335

336

337

338

339

340

341

utility and future perspectives. World Journal of Clinical Cases, 6(15):6-18.

34. Barbagallo C, Brex D, Caponnetto A, Cirnigliaro M, Scalia M, Magnano A, Caltabiano R, Barbagallo D, Biondi A, Cappellani A, Basile F, Di Pietro C, Purrello M, Ragusa M. LncRNA UCA1, Upregulated in CRC Biopsies and Downregulated in Serum Exosomes, Controls mRNA Expression by RNA-RNA Interactions. Molecular Therapy - Nucleic Acids, 2018, 12:229-241. 
Figure 1

Figure 1: The mRNA expression level of E2F1, E2F2, E2F3, E2F4, E2F5, E2F6, E2F7 and E2F8. (A) E2F1; (B) E2F2; (C) E2F3; (D) E2F4; (E) E2F5; (F) E2F6; (G) E2F7 and (H)E2F8;

Figure 1: The mRNA expression level of E2F1, E2F2, E2F3, E2F4, E2F5, E2F6, E2F7 and E2F8. (A) E2F1; (B) E2F2; (C) E2F3; (D) E2F4; (E) E2F5; (F) E2F6; (G) E2F7 and (H)E2F8;
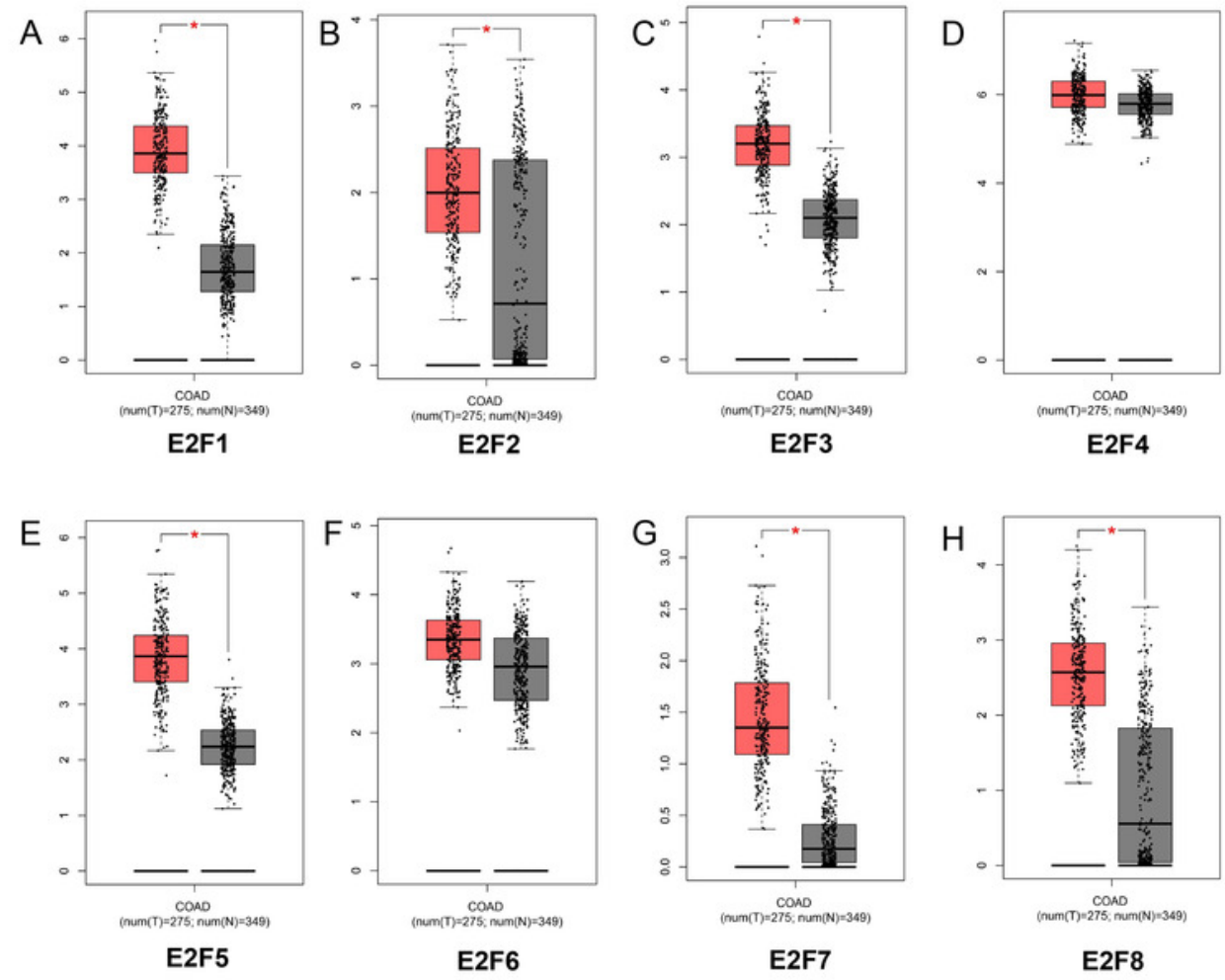
Figure 2

Figure 2: Transcripts per million (TPM) of E2F1, E2F2, E2F3, E2F4, E2F5, E2F6, E2F7 and E2F8. (A) E2F1; (B) E2F2; (C) E2F3; (D) E2F4; (E) E2F5; (F) E2F6; (G) E2F7 and (H) E2F8D

Figure 2: Transcripts per million (TPM) of E2F1, E2F2, E2F3, E2F4, E2F5, E2F6, E2F7 and E2F8. (A) E2F1; (B) E2F2; (C) E2F3; (D) E2F4; (E) E2F5; (F) E2F6; (G) E2F7 and (H) E2F8D

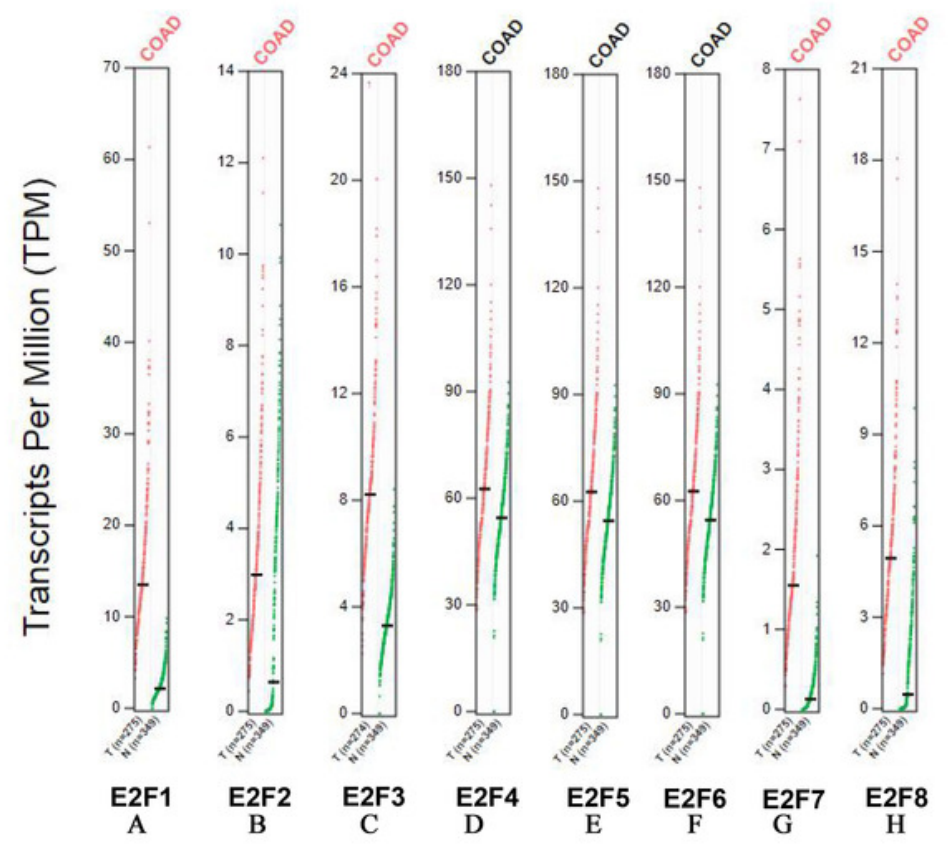


Figure 3

Figure 3: The relationship between the level of E2F genes and tumor stages in patients with colon cancer. (A) E2F1; (B) E2F2; (C) E2F3; (D) E2F4; (E) E2F5; (F) E2F6; (G) E2F7 and $(\mathrm{H}) \mathrm{E} 2 \mathrm{~F} 8 \mathrm{\square}$

Figure 3: The relationship between the level of E2F genes and tumor stages in patients with colon cancer. (A) E2F1; (B) E2F2; (C) E2F3; (D) E2F4; (E) E2F5; (F) E2F6; (G) E2F7 and (H) E2F8—

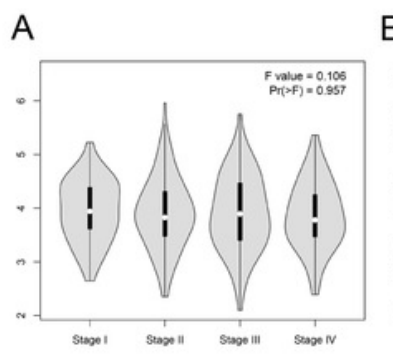

E2F1

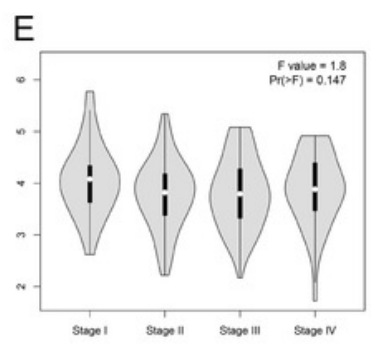

E2F5

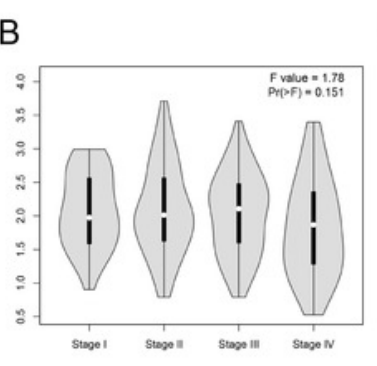

E2F2
C

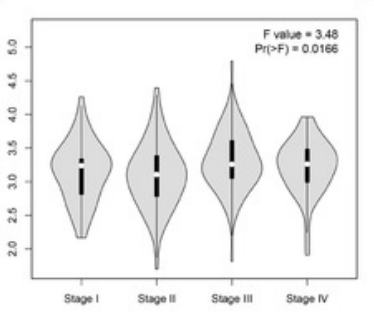

E2F3
D

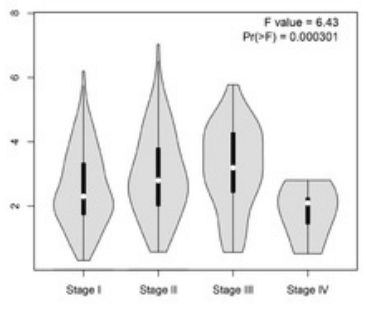

E2F4

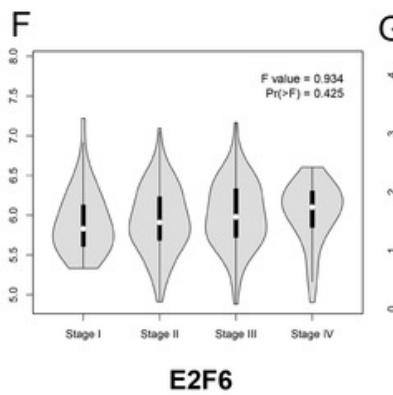

G

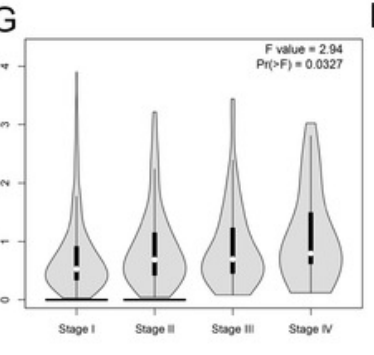

E2F7

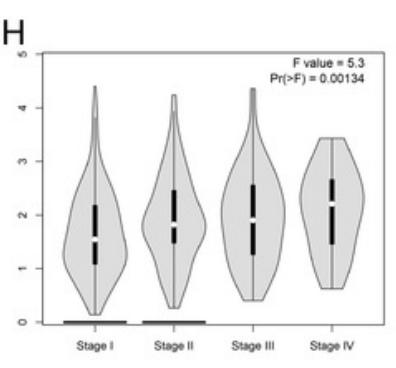

E2F8 
Figure 4

Figure 4: The relationship between the level of E2F genes and overall survival in patients with colon cancer. (A) E2F1; (B) E2F2; (C) E2F3; (D) E2F4; (E) E2F5; (F) E2F6; (G) E2F7 and (H) E2F8马

Figure 4: The relationship between the level of E2F genes and overall survival in patients with colon cancer. (A) E2F1; (B) E2F2; (C) E2F3; (D) E2F4; (E) E2F5; (F) E2F6; (G) E2F7 and (H) E2F8ロ

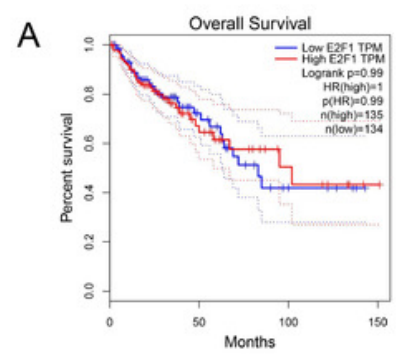

E2F1

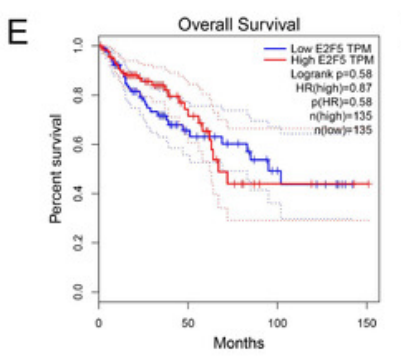

E2F5

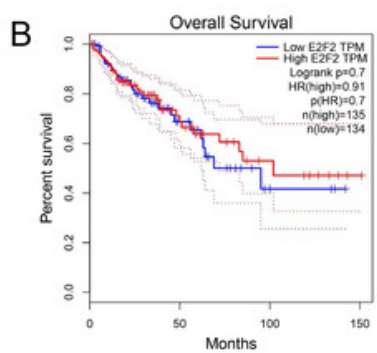

E2F2

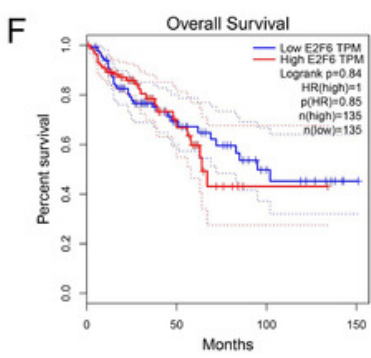

E2F6

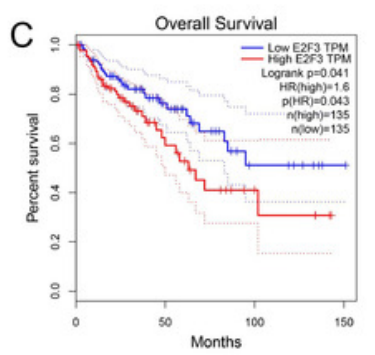

E2F3

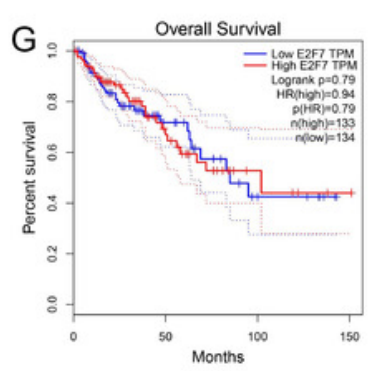

E2F7

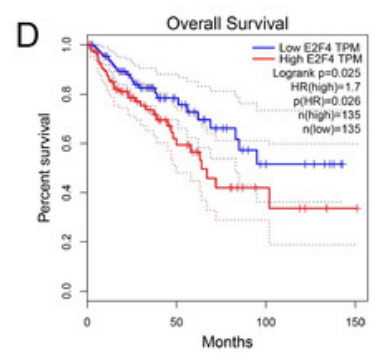

E2F4

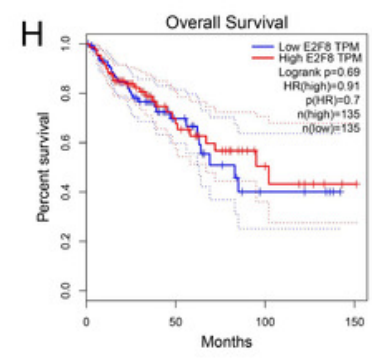

E2F8 
Figure 5

Figure 5: The immunohistochemical staining data obtained from HPA database

Figure 5: The immunohistochemical staining data obtained from HPA database
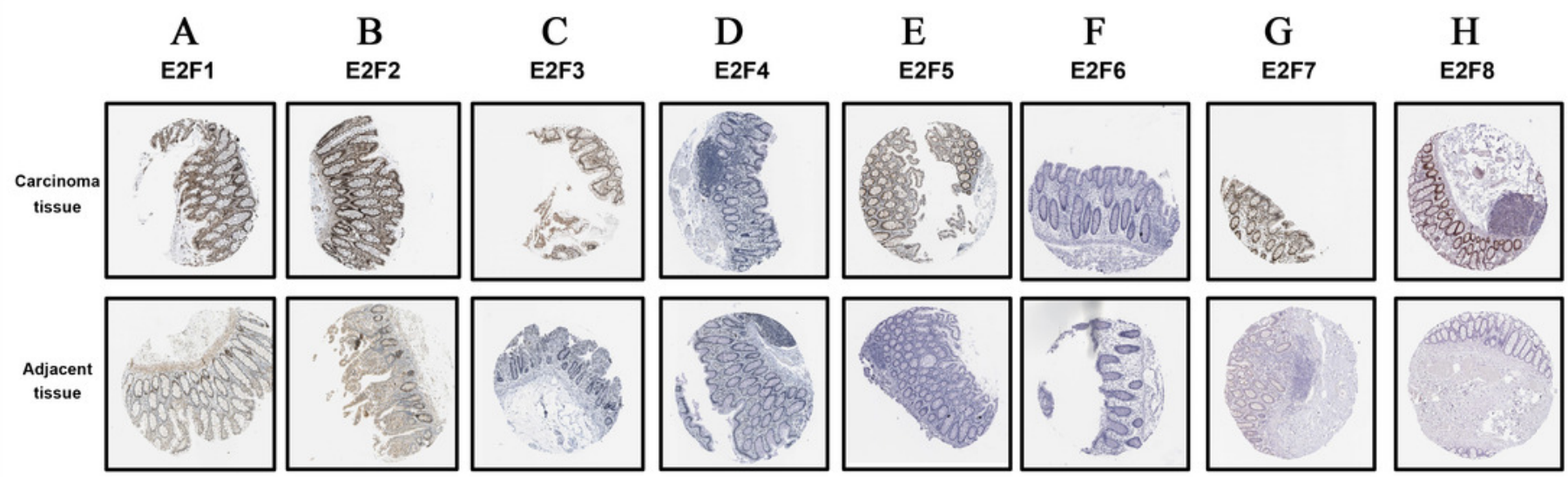

I

G

K

$\mathrm{L}$

M

$\mathrm{N}$

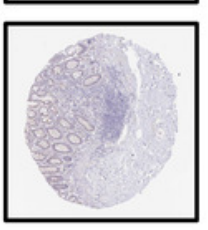

O

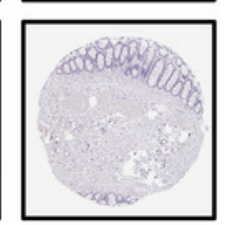

$\mathbf{P}$ 
Figure 6

Figure 6: Validation of E2F genes in the carcinoma tissues and adjacent tissues from patients with colon cancer using real time PCR. ${ }^{*} P<0.05$ vs carcinoma tissue.

Figure 6: Validation of E2F genes in the carcinoma tissues and adjacent tissues from patients with colon cancer using real time PCR. $* \mathrm{P}<0.05$ vs carcinoma tissue.

Adjacent tissue

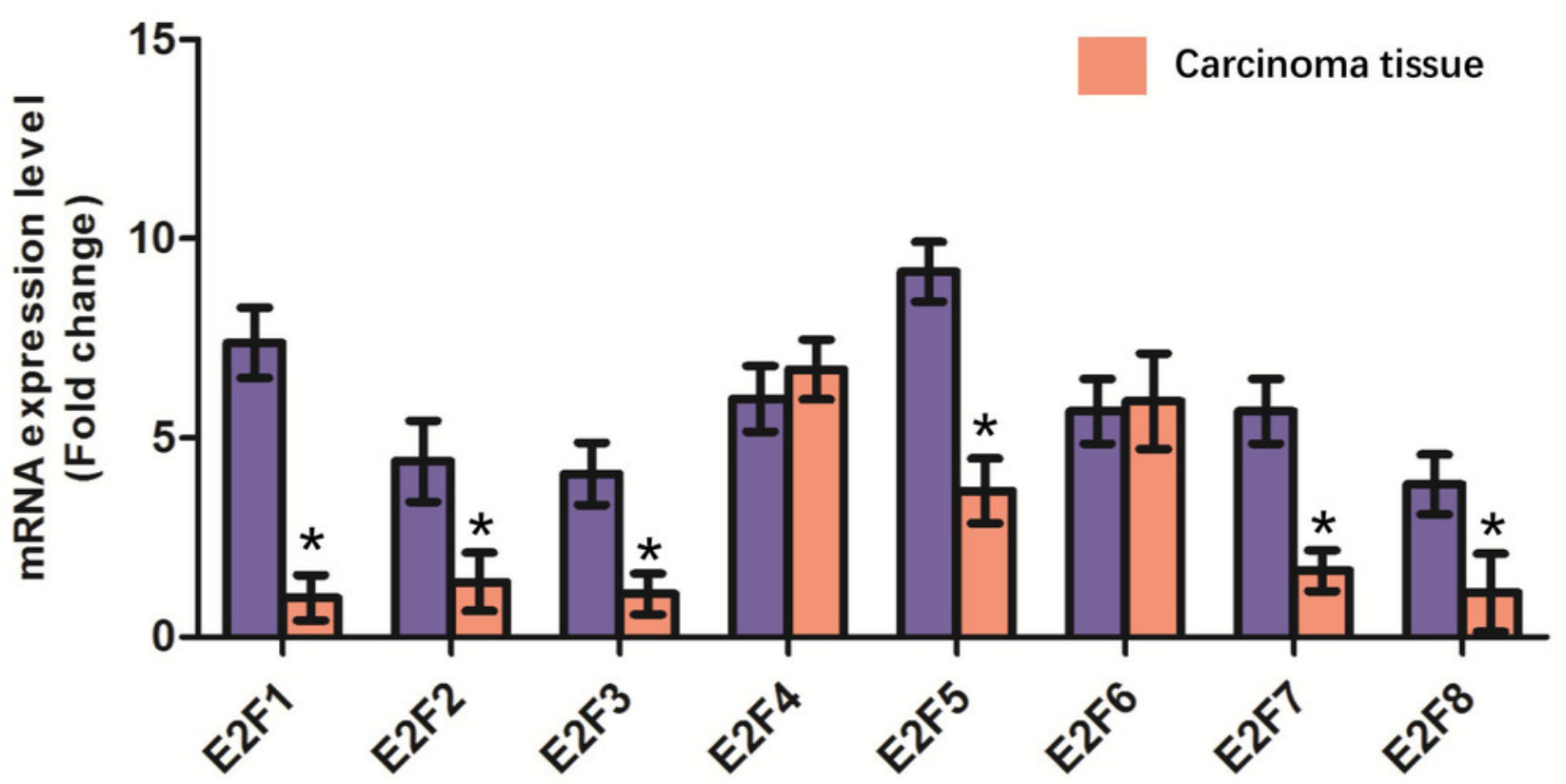




\section{Table 1 (on next page)}

Table 1. Primer sequences used in this study

Table 1. Primer sequences used in this study 
2

\begin{tabular}{llll}
\hline Gene & Species & Forward & Reverse \\
\hline E2F1 & Human & AGCATGATCCGAGATGTGGAA & TGCTCGCACGATCGTAGCCCT \\
E2F2 & Human & ACGATGTCGATGCTAGCGTGG & CGTCGTACCCAACTGCTAGCT \\
E2F3 & Human & ACGTCGTAGCTGATGGGCAGT & CGGTGTACGTACCAAAACTG \\
E2F4 & Human & ACAAATGCATGGGTCCGTCGA & GACATGCCGCCTGGAGAAAC \\
E2F5 & Human & ACGTGGACTGGCCCAACTGCC & GACATGCCGCCTGGAGAAAC \\
E2F6 & Human & CGCGTAGCTACGCTACAGCTAC & ACGTGATCGTAGCTGATCGCC \\
E2F7 & Human & CACACACGTTAAACACCAACCT & CGTGTGGGGCACGTGGCAAC \\
E2F8 & Human & ACAAAGTGCGGTCACGTTTCAT & ACGATCGATGCTGATCGCGA \\
\hline
\end{tabular}

3 


\section{Table 2 (on next page)}

Table 2: Gene ontology analysis of E2F family associated with colon cancer

Table 2: Gene ontology analysis of E2F family associated with colon cancer 
Table 2: Gene ontology analysis of E2F family associated with colon cancer

2

\begin{tabular}{|c|c|c|c|c|c|c|}
\hline Category & Term & Count & $\%$ & P-Value & FDR & Genes \\
\hline GOTERM_BP_DIRECT & GO:0006351 transcription, DNA-templated & 6 & 0.423429781 & $6.74 \mathrm{E}-09$ & $5.53 \mathrm{E}-06$ & E2F3, E2F4, E2F5, E2F6, E2F7, E2F8 \\
\hline GOTERM_BP_DIRECT & GO:0032877 positive regulation of DNA endoreduplication & 2 & 0.14114326 & 8.11E-04 & 0.663544451 & $\mathrm{E} 2 \mathrm{~F} 7, \mathrm{E} 2 \mathrm{~F} 8$ \\
\hline GOTERM_BP_DIRECT & GO:0070365 hepatocyte differentiation & 2 & 0.14114326 & 0.001622454 & 1.322792672 & $\mathrm{E} 2 \mathrm{~F} 7, \mathrm{E} 2 \mathrm{~F} 8$ \\
\hline GOTERM_BP_DIRECT & GO:0032466 negative regulation of cytokinesis & 2 & 0.14114326 & 0.002027739 & 1.65081418 & E2F7, E2F8 \\
\hline GOTERM_BP_DIRECT & GO:0060718 chorionic trophoblast cell differentiation & 2 & 0.14114326 & 0.002432892 & 1.977771789 & $\mathrm{E} 2 \mathrm{~F} 7, \mathrm{E} 2 \mathrm{~F} 8$ \\
\hline GOTERM_CC_DIRECT & GO:0005667 transcription factor complex & 6 & 0.423429781 & $1.61 \mathrm{E}-10$ & $8.27739543574068 \mathrm{E}-08$ & $\mathrm{E} 2 \mathrm{~F} 3, \mathrm{E} 2 \mathrm{~F} 4, \mathrm{E} 2 \mathrm{~F} 5, \mathrm{E} 2 \mathrm{~F} 6, \mathrm{E} 2 \mathrm{~F} 7, \mathrm{E} 2 \mathrm{~F} 8$ \\
\hline GOTERM_MF_DIRECT & GO:0001047 core promoter binding & 3 & 0.211714891 & $1.15 \mathrm{E}-04$ & 0.068932147 & $\mathrm{E} 2 \mathrm{~F} 3, \mathrm{E} 2 \mathrm{~F} 7, \mathrm{E} 2 \mathrm{~F} 8$ \\
\hline GOTERM_MF_DIRECT & $\begin{array}{l}\text { GO:0003700 -ranscription factor activity, sequence-specific DNA } \\
\text { binding }\end{array}$ & 4 & 0.282286521 & $4.73 \mathrm{E}-04$ & 0.283673732 & E2F3, E2F4, E2F5, E2F6 \\
\hline GOTERM_MF_DIRECT & GO:0003677 DNA binding & 3 & 0.211714891 & 0.016757148 & 9.649556019 & $\mathrm{E} 2 \mathrm{~F} 4, \mathrm{E} 2 \mathrm{~F} 5, \mathrm{E} 2 \mathrm{~F} 6$ \\
\hline GOTERM_MF_DIRECT & GO:0003714 transcription corepressor activity & 2 & 0.14114326 & 0.04355609 & 23.46393428 & $\mathrm{E} 2 \mathrm{~F} 7, \mathrm{E} 2 \mathrm{~F} 8$ \\
\hline
\end{tabular}

3 


\section{Table 3(on next page)}

Table 3: KEGG pathway analysis of E2F family associated with colon cancer

Table 3: KEGG pathway analysis of E2F family associated with colon cancer 
Table 3: KEGG pathway analysis of E2F family associated with colon cancer

\begin{tabular}{|c|c|c|c|c|c|c|}
\hline Category & Term & Count & $\%$ & P-Value & FDR & Genes \\
\hline KEGG_PATHWAY & cfa04110:Cell cycle & 5 & 0.352858151 & $1.05 \mathrm{E}-07$ & 7.14E-05 & $\mathrm{E} 2 \mathrm{~F} 1, \mathrm{E} 2 \mathrm{~F} 2, \mathrm{E} 2 \mathrm{~F} 3, \mathrm{E} 2 \mathrm{~F} 4, \mathrm{E} 2 \mathrm{~F} 5$ \\
\hline KEGG_PATHWAY & cfa05219:Bladder cancer & 3 & 0.211714891 & $2.14 \mathrm{E}-04$ & 0.145507524 & $\mathrm{E} 2 \mathrm{~F} 1, \mathrm{E} 2 \mathrm{~F} 2, \mathrm{E} 2 \mathrm{~F} 3$ \\
\hline KEGG_PATHWAY & cfa05223:Non-small cell lung cancer & 3 & 0.211714891 & $4.16 \mathrm{E}-04$ & 0.282144998 & $\mathrm{E} 2 \mathrm{~F} 1, \mathrm{E} 2 \mathrm{~F} 2, \mathrm{E} 2 \mathrm{~F} 3$ \\
\hline KEGG_PATHWAY & cfa05212:Pancreatic cancer & 3 & 0.211714891 & $5.25 \mathrm{E}-04$ & 0.355786534 & $\mathrm{E} 2 \mathrm{~F} 1, \mathrm{E} 2 \mathrm{~F} 2, \mathrm{E} 2 \mathrm{~F} 3$ \\
\hline KEGG_PATHWAY & cfa05214:Glioma & 3 & 0.211714891 & $5.41 \mathrm{E}-04$ & 0.366990838 & $\mathrm{E} 2 \mathrm{~F} 1, \mathrm{E} 2 \mathrm{~F} 2, \mathrm{E} 2 \mathrm{~F} 3$ \\
\hline KEGG_PATHWAY & cfa05218:Melanoma & 3 & 0.211714891 & $6.46 \mathrm{E}-04$ & 0.437793075 & $\mathrm{E} 2 \mathrm{~F} 1, \mathrm{E} 2 \mathrm{~F} 2, \mathrm{E} 2 \mathrm{~F} 3$ \\
\hline KEGG_PATHWAY & cfa05220:Chronic myeloid leukemia & 3 & 0.211714891 & $6.64 \mathrm{E}-04$ & 0.450188061 & $\mathrm{E} 2 \mathrm{~F} 1, \mathrm{E} 2 \mathrm{~F} 2, \mathrm{E} 2 \mathrm{~F} 3$ \\
\hline KEGG_PATHWAY & cfa05222:Small cell lung cancer & 3 & 0.211714891 & $8.82 \mathrm{E}-04$ & 0.597682584 & $\mathrm{E} 2 \mathrm{~F} 1, \mathrm{E} 2 \mathrm{~F} 2, \mathrm{E} 2 \mathrm{~F} 3$ \\
\hline KEGG_PATHWAY & cfa05215:Prostate cancer & 3 & 0.211714891 & $9.69 \mathrm{E}-04$ & 0.656358939 & $\mathrm{E} 2 \mathrm{~F} 1, \mathrm{E} 2 \mathrm{~F} 2, \mathrm{E} 2 \mathrm{~F} 3$ \\
\hline
\end{tabular}


